\title{
Cosmic flow around local massive galaxies
}

\author{
Olga G. Kashibadze ${ }^{\star}$ and Igor D. Karachentsev
}

\begin{abstract}
Special Astrophysical Observatory of the Russian Academy of Sciences Nizhnij Arkhyz, 369167 Karachay-Cherkessia, Russia e-mail: phiruzi@gmail.com
\end{abstract}

Received 24 July 2017 / Accepted 19 September 2017

\begin{abstract}
Aims. We use accurate data on distances and radial velocities of galaxies around the Local Group, as well as around 14 other massive nearby groups, to estimate their radius of the zero-velocity surface, $R_{0}$, which separates any group against the global cosmic expansion Methods. Our $R_{0}$ estimate was based on fitting the data to the velocity field expected from the spherical infall model, including effects of the cosmological constant. The reported uncertainties were derived by a Monte Carlo simulation.

Results. Testing various assumptions about a location of the group barycentre, we found the optimal estimates of the radius to be $0.91 \pm 0.05 \mathrm{Mpc}$ for the Local Group, and $0.93 \pm 0.02 \mathrm{Mpc}$ for a synthetic group stacked from 14 other groups in the Local Volume. Under the standard Planck model parameters, these quantities correspond to the total mass of the group $\sim(1.6 \pm 0.2) \times 10^{12} M_{\odot}$. Thus, we are faced with the paradoxical result that the total mass estimate on the scale of $R_{0} \approx(3-4) R_{\text {vir }}$ is only $60 \%$ of the virial mass estimate. Anyway, we conclude that wide outskirts of the nearby groups do not contain a large amount of hidden mass outside their virial radius.
\end{abstract}

Key words. galaxies: groups: general - galaxies: groups: individual: Local Group

\section{Introduction}

Any overdense region in the Universe is driven by the competition between its self-gravity and the cosmic expansion, and therefore can be characterized by an idealized zero-velocity surface that separates these zones. de Vaucouleurs (1958, 1964, 1972) presupposed systematic deviations from linearity in the velocity-distance relation and interpreted these deviations as a local phenomenon caused by the Virgo complex. The expected effect has only subsequently been supported by observations. Peebles (1976) found the virgocentric infall signal using the field galaxy data available at that time (Sandage \& Tammann 1975).

Lynden-Bell (1981) and Sandage (1986) focussed on the Local Group of galaxies. They showed that, in the simplest case of the spherically symmetric system in the empty Universe with $\Lambda=0$, the radius of the zero-velocity surface $R_{0}$ and the total mass of the group $M_{T}^{0}$ are related as

$M_{T}^{0}=\left(\pi^{2} / 8 G\right) \times R_{0}^{3} \times T_{0}^{-2}$,

where $G$ is the gravitational constant and $T_{0}$ is the age of the Universe (Lynden-Bell 1981; Sandage 1986). In the standard cosmological $\Lambda$ CDM model, where $\Omega_{\mathrm{m}}$ is the mean cosmic density of matter and $H_{0}$ the Hubble parameter, the relation between $R_{0}$ and $M_{T}$ becomes

$M_{T}=\left(\pi^{2} / 8 G\right) \times R_{0}^{3} \times H_{0}^{2} / f^{2}\left(\Omega_{\mathrm{m}}\right)$,

where the dimensionless parameter

$f\left(\Omega_{\mathrm{m}}\right)=\left(1-\Omega_{\mathrm{m}}\right)^{-1}-\frac{\Omega_{\mathrm{m}}}{2}\left(1-\Omega_{\mathrm{m}}\right)^{-\frac{3}{2}} \cosh ^{-1}\left(\frac{2}{\Omega_{\mathrm{m}}}-1\right)$

changes in the range from 1 to $2 / 3$ while varying $\Omega_{\mathrm{m}}$ from 0 to 1 . Taking the Planck model parameters $\Omega_{\mathrm{m}}=0.315, \Omega_{\lambda}=0.685$

\footnotetext{
$\star$ F.k.a. Nasonova.
}

and $H_{0}=67.3 \mathrm{~km} \mathrm{~s}^{-1} \mathrm{Mpc}^{-1}$ (Planck Collaboration XVI 2014), we obtain the relation

$\left(M_{T} / M_{\odot}\right)_{0.315}=1.95 \times 10^{12}\left(R_{0} / \mathrm{Mpc}\right)^{3}$,

which is by 1.50 times more than the classical estimate from Eq. (1).

This method was sucessfully applied to determine masses of the Local Group (Ekholm et al. 2001; Karachentsev et al. 2002, 2009; Teerikorpi et al. 2005), M81 group (Karachentsev \& Kashibadze 2006), CenA group (Karachentsev et al. 2006), as well as the Virgo cluster (Tully \& Shaya 1984; Karachentsev \& Nasonova 2010; Karachentsev et al. 2014) and the Fornax cluster (Nasonova et al. 2011).

It is important to stress that the $R_{0}$ method estimates the total mass of a group independently of mass estimates based on virial motions. Notably, the corresponding total mass $M_{T}$ is confined on the linear scale of $R_{0}$, which is three to four times as large as the virial radius of a group or cluster, $R_{\text {vir }}$.

The implementation of the $R_{0}$ method became possible with wholesale measurements of distances to nearby galaxies from luminosities of the red giant branch stars (TRGB) with accuracy of $\sim 5 \%$ attainable by the Hubble Space Telescope. In the Local Volume, limited to $11 \mathrm{Mpc}$, there are about a thousand known galaxies; most of these galaxies have measured radial velocities with a typical accuracy less than $5 \mathrm{~km} \mathrm{~s}^{-1}$. About onethird of the Local Volume population already has accurate TRGB distance estimates. The compilation of observational data on these objects is presented in the Updated Nearby Galaxy Catalog (Karachentsev et al. 2013) and its latest electronic version ${ }^{1}$ (Kaisina et al. 2012). For a typical galaxy of the Local Volume with a distance of $\sim 6 \mathrm{Mpc}$, the TRGB distance error of $\sim 300 \mathrm{kpc}$ is comparable with a virial radius of the group, thus its location can be confidently fixed relative to the group centroid and

1 http://sao.ru/lv/lvgdb/ 


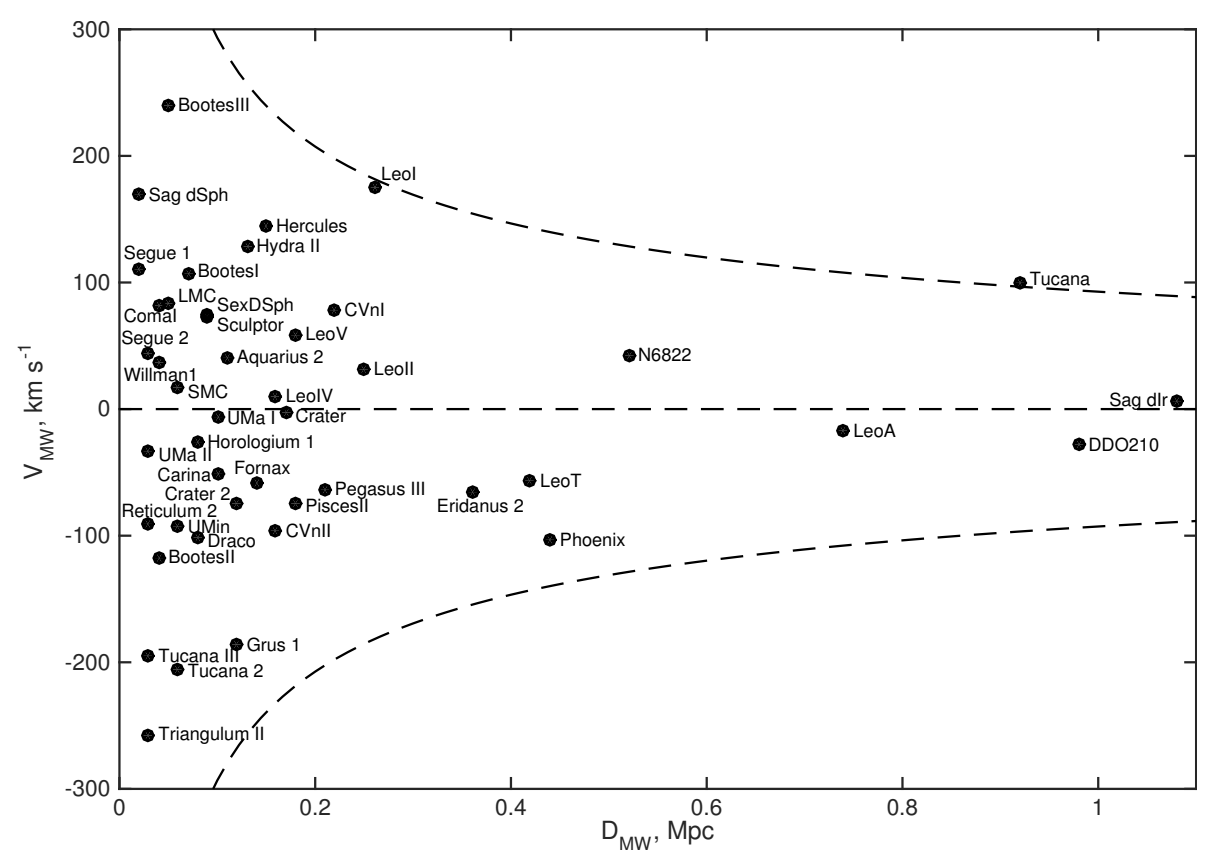

Fig. 1. Distribution of 45 Milky Way satellites by their spatial distances and radial velocities relative to the Milky Way. Dashed lines correspond to the parabolic velocity for a point mass of $1 \times 10^{12} M_{\odot}$. zero velocity surface. Other methods of secondary importance are the Tully \& Fisher (1977) relation distances or the brightest stars distances with an accuracy of $\sim(20-30) \%$, which do not provide an opportunity to determine $R_{0}$ value even for the nearest groups.

Below we use the most complete data on distances and radial velocities of the Local Volume galaxies to estimate the zerovelocity radius around the local massive galaxies.

\section{Galaxy motions around the Milky Way and M31}

The recent surveys of large sky areas (Abazajian et al. 2009; Tonry et al. 2012; Koposov et al. 2015) led to the discovery of new Milky Way (MW) dwarf satellites with low luminosities and extremely low surface brightnesses. The recent overview by McConnachie (2012) reports 29 MW satellites with measured radial velocities and accurate distances. In recent years, this list has been expanded up to 45 objects. The corresponding data are presented in Table 1 . The table columns contain (1) galaxy name; (2) equatorial coordinates J2000.0; and (3) tidal index,

$T I=\max \left[\log \left(M_{n}^{*} / D_{n}^{3}\right)\right]-10.96, \quad n=1,2, \ldots N$,

distinguishing the most significant galaxy (main disturber = MD) among $N$ neighbouring galaxies, whose tidal force dominates the remaining galaxies with masses $M_{n}^{*}$ and spatial separations $D_{n}$. The constant, -10.96 , is chosen in such a way that $T I=0$ corresponds to a significant neighbour located on the zero velocity surface with $T I<0$ galaxies ranked as isolated. Finally, Col. (4) lists the main disturber name, Col. (5) distance to a galaxy in Mpc, and Cols. $(6,7)$ radial velocity of a galaxy (in $\mathrm{km} \mathrm{s}^{-1}$ ) relative to the Sun and relative to the MW centre with apex parameters adopted in NASA Extragalactic Database (NED). References to the used values of distances and velocities of galaxies are presented in the Local Volume Galaxies Database $^{2}$.

The distribution of 45 satellites of the MW by their Galactocentric distances and radial velocities is shown in Fig. 1. The dashed lines correspond to the parabolic velocity for a point mass

\footnotetext{
2 http://sao.ru/lv/lvgdb/
}

of $1 \times 10^{12} M_{\odot}$. The velocity distribution of satellites looks symmetrical relative to the MW centre, although two satellites with near-parabolic velocities - Tucana and LeoI - are close to the upper escape limit. Three MW satellites, Sag dIr, DDO 210, and Tucana with distances $D \sim 1 \mathrm{Mpc}$ and negative $\Theta_{1}$, belong to field galaxies. However, the MW is dynamically the most significant neighbour for each of these.

Specialized searches for faint satellites in the outskirts of the spiral galaxy M31 in the Andromeda constellation (Ibata et al. 2007, 2014; Martin et al. 2009) has proved to be notably productive. While the sample by McConnachie (2012) included 23 satellites, now their number is roughly doubled amounting up to 44. The data on these satellites are presented in Table 2, where the first six columns have the same meaning as in Table 1 . The seventh column of Table 2 contains spatial distances of satellites (in Mpc) relative to M31, while eighth and ninth list the projected separation of satellites in the sky (in Mpc) and their differential radial velocities relative to M31 (in $\mathrm{km} \mathrm{s}^{-1}$ ). Aside from dwarf galaxies, we tabulate also the data on eight distant globular clusters from PAndAS survey (Huxor et al. 2014) with measured radial velocities. Their spatial distances still remain unknown, and we set them equal to $0.78 \mathrm{Mpc}$.

The distribution of $44+8$ test particles by their differential radial velocities and projected separations relative to $M 31$ is presented in Fig. 2. The dashed lines also mean the parabolic velocity for a point mass of $1 \times 10^{12} M_{\odot}$. Similar to the MW case, the distribution of M31 satellites by their relative velocities seems to be very symmetrical; two satellites - And XIV and And XII have near-parabolic velocities that are close to the lower escape limit.

\section{Orbital masses of the Milky Way and M31}

For a massive galaxy surrounded by small satellites, the orbital mass estimate is expressed as

$M_{\text {orb }}=(32 / 3 \pi) \times\left(1-2 e^{2} / 3\right)^{-1} \times G^{-1} \times\left\langle\Delta V^{2} \times R_{p}\right\rangle$,

where $\left\langle\Delta V^{2} \times R_{p}\right\rangle$ is the mean product of squared radial velocity difference of a satellite with its projected distance from the main galaxy and $e$ is the orbit eccentricity (Karachentsev \& Kudrya 2014). This relation is obtained under 
Table 1. Milky Way companions with $T I>-0.5$.

\begin{tabular}{|c|c|c|c|c|c|c|}
\hline Name & $\begin{array}{c}\text { RA (2000.0) Dec } \\
\text { hh mm ss dd mm ss }\end{array}$ & $T I$ & MD & $\begin{array}{l}D_{\mathrm{MW}} \\
\mathrm{Mpc}\end{array}$ & $\begin{array}{r}V_{\mathrm{h}} \\
\mathrm{km} \mathrm{s}^{-1}\end{array}$ & $\begin{array}{r}V_{\mathrm{MW}} \\
\mathrm{km} \mathrm{s}^{-1}\end{array}$ \\
\hline (1) & (2) & (3) & (4) & (5) & (6) & (7) \\
\hline SMC & $005238.0-724801$ & 3.32 & LMC & 0.06 & 158 & 17 \\
\hline Sculptor & $010009.4-334233$ & 2.79 & MWay & 0.09 & 105 & 72 \\
\hline Phoenix & $015106.3-442641$ & 0.73 & MWay & 0.44 & -13 & -103 \\
\hline Triangulum II & $021317.4+361042$ & 3.97 & MWay & 0.03 & -382 & -257 \\
\hline Segue 2 & $021916.0+201031$ & 3.83 & MWay & 0.03 & -39 & 44 \\
\hline Fornax & $023954.7-343133$ & 2.19 & MWay & 0.14 & 29 & -59 \\
\hline Horologium 1 & $025531.7-540708$ & 2.94 & MWay & 0.08 & 113 & -26 \\
\hline Reticulum 2 & $033542.1-540257$ & 4.15 & MWay & 0.03 & 64 & -91 \\
\hline Eridanus 2 & $034421.1-433159$ & 1.00 & MWay & 0.36 & 76 & -66 \\
\hline LMC & $052334.6-694522$ & 3.56 & MWay & 0.05 & 278 & 84 \\
\hline Carina & $064136.7-505758$ & 2.63 & MWay & 0.10 & 224 & -52 \\
\hline UMa II & $085130.0+630748$ & 3.92 & MWay & 0.03 & -116 & -33 \\
\hline Leo T & $093453.4+170305$ & 0.77 & MWay & 0.42 & 39 & -57 \\
\hline Leo A & $095926.4+304447$ & 0.03 & MWay & 0.74 & 24 & -17 \\
\hline Segue 1 & $100703.2+160425$ & 4.32 & MWay & 0.02 & 206 & 111 \\
\hline Leo I & $100826.9+121829$ & 1.37 & MWay & 0.26 & 283 & 175 \\
\hline Sex dSph & $101303.0-013652$ & 2.74 & MWay & 0.09 & 227 & 75 \\
\hline UMa I & $103452.8+515512$ & 2.59 & MWay & 0.10 & -55 & -7 \\
\hline Willman 1 & $104921.0+510300$ & 3.76 & MWay & 0.04 & -12 & 36 \\
\hline Leo II & $111329.2+220917$ & 1.45 & MWay & 0.25 & 86 & 32 \\
\hline Leo V & $113109.6+021312$ & 1.89 & MWay & 0.18 & 173 & 59 \\
\hline Leo IV & $113257.0-003200$ & 2.05 & MWay & 0.16 & 132 & 10 \\
\hline Crater & $113615.8-105240$ & 1.96 & MWay & 0.17 & 148 & -2 \\
\hline Crater 2 & $114914.4-182447$ & 2.50 & MWay & 0.12 & 88 & -74 \\
\hline Hydra II & $122142.1-315907$ & 2.29 & MWay & 0.13 & 303 & 129 \\
\hline Coma I & $122659.0+235415$ & 3.74 & MWay & 0.04 & 98 & 82 \\
\hline CVn II & $125710.0+341915$ & 2.04 & MWay & 0.16 & -129 & -96 \\
\hline CVn I & $132803.5+333321$ & 1.60 & MWay & 0.22 & 31 & 78 \\
\hline Bootes III & $135707.4+264630$ & 3.67 & MWay & 0.05 & 198 & 240 \\
\hline Bootes II & $135800.0+125100$ & 3.86 & MWay & 0.04 & -117 & -117 \\
\hline Bootes I & $140000.0+143000$ & 3.25 & MWay & 0.07 & 99 & 106 \\
\hline UMin & $150911.3+671252$ & 3.27 & MWay & 0.06 & -255 & -93 \\
\hline Hercules & $163102.0+124730$ & 2.19 & MWay & 0.15 & 45 & 145 \\
\hline Draco & $172001.4+575434$ & 2.94 & MWay & 0.08 & -296 & -101 \\
\hline Sag dSph & $185503.1-302842$ & 5.36 & MWay & 0.02 & 140 & 169 \\
\hline Sag dIr & $192959.0-174041$ & -0.44 & MWay & 1.08 & -79 & 7 \\
\hline NGC 6822 & $194457.7-144811$ & 0.52 & MWay & 0.52 & -57 & 43 \\
\hline DDO 210 & $204651.8-125053$ & -0.31 & MWay & 0.98 & -140 & -28 \\
\hline Pegasus III & $222424.2+052436$ & 1.73 & MWay & 0.21 & -223 & -63 \\
\hline Aquarius 2 & $223355.5-091939$ & 2.56 & MWay & 0.11 & -71 & 41 \\
\hline Tucana & $224149.0-642512$ & -0.24 & MWay & 0.92 & 194 & 99 \\
\hline Tucana 2 & $225155.1-583408$ & 3.46 & MWay & 0.06 & -129 & -205 \\
\hline Grus 1 & $225642.4-500948$ & 2.45 & MWay & 0.12 & -140 & -186 \\
\hline Pisces II & $225831.0+055709$ & 1.88 & MWay & 0.18 & -226 & -75 \\
\hline Tucana III & $235636.0-593600$ & 3.90 & MWay & 0.03 & -102 & -195 \\
\hline
\end{tabular}

the assumption of uniformly random orientation of satellite orbits relative to the line of sight. With the typical eccentricity value of $\left\langle e^{2}\right\rangle=1 / 2$ (Barber et al. 2014) the relation (6) becomes

$M_{\text {orb }}=(16 / \pi) \times G^{-1} \times\left\langle\Delta V^{2} \times R_{p}\right\rangle$.

Applying Eq. (7) to the assembly of the MW and M31 satellites, we get values for orbital masses $M_{\text {orb }}(\mathrm{MW})=1.51 \times 10^{12} M_{\odot}$ and $M_{\text {orb }}(\mathrm{M} 31)=1.69 \times 10^{12} M_{\odot}$. Since in the case of MW satellites we observe their $3 \mathrm{D}$ distances, then project distances, the orbital mass estimation should be reduced by a factor of $(\pi / 4)$ yielding $M_{\text {orb }}(\mathrm{MW})=1.18 \times 10^{12} M_{\odot}$. Hence, the ratio of mass estimates for these two galaxies reaches

$M_{\text {orb }}(\mathrm{MW}) / M_{\text {orb }}(\mathrm{M} 31) \simeq 0.70$.

This value is quite close to the ratio $M_{\text {orb }}(\mathrm{MW}) / M_{\text {orb }}(\mathrm{M} 31)=$ 0.82 obtained by Karachentsev \& Kashibadze (2006) from a minimum value for scatter of peculiar velocities with respect to the Hubble regression line, while varying the centroid position between the MW and M31.

A comparison of the derived total masses of the MW and M31, their combined mass, and the mass ratio with other mass estimates in the recent literature is presented in Table 3. These estimates were based on kinematics of satellites and globular clusters assuming that the MW and M31 haloes follow the standard NFW profile or fit the kinematics of high-velocity stars and blue horizontal branch stars. Our present measurements are in good agreement with the median values given in the last line of Table 3. An essential part of the mismatch between the different estimates in Table 3 may arise from the observed orbital anisotropy of the MW and M31 satellites (Ibata et al. 2013; Pawlowski et al. 2014) and from the uncertain dynamical status of two MW satellites, Leo I and Tucana, and the two 
Table 2. M31 companions.

\begin{tabular}{|c|c|c|c|c|c|c|c|c|}
\hline Name & $\begin{array}{c}\text { RA (2000.0) Dec } \\
\text { hh mm ss dd mm ss }\end{array}$ & $T I$ & MD & $\begin{array}{l}D_{\mathrm{MW}} \\
\mathrm{Mpc}\end{array}$ & $\begin{array}{r}V_{\mathrm{h}} \\
\mathrm{km} \mathrm{s}^{-1}\end{array}$ & $\begin{array}{r}D_{\mathrm{M} 31} \\
\mathrm{Mpc} \\
\end{array}$ & $\begin{array}{c}R_{\mathrm{p}} \\
\mathrm{Mpc}\end{array}$ & $\begin{array}{c}\Delta V \\
\mathrm{~km} \mathrm{~s}^{-1}\end{array}$ \\
\hline (1) & (2) & (3) & (4) & $(5)$ & (6) & $(7)$ & $(8)$ & (9) \\
\hline WLM & $000158.1-152740$ & -0.01 & M31 & 0.98 & -122 & 0.88 & 0.75 & 14 \\
\hline And XVIII & $000214.5+450520$ & 0.72 & M31 & 1.31 & -332 & 0.51 & 0.11 & -14 \\
\hline And XIX & $001932.1+350237$ & 2.21 & M31 & 0.93 & -111 & 0.16 & 0.10 & 187 \\
\hline IC 10 & $002024.5+591730$ & 1.58 & M31 & 0.79 & -346 & 0.26 & 0.25 & -32 \\
\hline And XXVI & $002345.6+475458$ & 2.58 & M31 & 0.76 & -261 & 0.12 & 0.10 & 50 \\
\hline Cetus & $002611.0-110240$ & 0.27 & M31 & 0.79 & -87 & 0.71 & 0.69 & 55 \\
\hline And XXV & $003008.9+465107$ & 3.01 & M31 & 0.81 & -108 & 0.09 & 0.08 & 200 \\
\hline NGC 147 & $003311.6+483028$ & 2.55 & M31 & 0.76 & -193 & 0.12 & 0.10 & 115 \\
\hline And III & $003533.8+362952$ & 2.80 & M31 & 0.75 & -346 & 0.10 & 0.07 & -54 \\
\hline Cas III & $003559.4+513335$ & 2.28 & M31 & 0.78 & -372 & 0.15 & 0.14 & -64 \\
\hline And $X X X$ & $003634.9+493848$ & 2.28 & NGC 185 & 0.68 & -141 & 0.18 & 0.11 & 166 \\
\hline And XVII & $003707.0+441920$ & 2.95 & M31 & 0.74 & -251 & 0.09 & 0.04 & 51 \\
\hline And XXVII & $003727.1+452313$ & 3.47 & M31 & 0.83 & -535 & 0.06 & 0.06 & -232 \\
\hline NGC 185 & $003858.0+482010$ & 2.03 & M31 & 0.66 & -203 & 0.18 & 0.10 & 102 \\
\hline NGC 205 & $004022.5+414111$ & 4.68 & M31 & 0.80 & -221 & 0.02 & 0.01 & 77 \\
\hline M 32 & $004242.1+405159$ & 4.38 & M31 & 0.79 & -202 & 0.03 & 0.01 & 93 \\
\hline M31 & $004244.5+411609$ & 2.79 & NGC 205 & 0.78 & -296 & 0.00 & 0.00 & 0 \\
\hline And I & $004540.0+380214$ & 2.77 & M31 & 0.73 & -376 & 0.10 & 0.04 & -86 \\
\hline And XI & $004620.0+334805$ & 2.43 & M31 & 0.73 & -419 & 0.14 & 0.10 & -137 \\
\hline And XII & $004727.0+342229$ & 2.82 & M31 & 0.83 & -556 & 0.10 & 0.09 & -274 \\
\hline Bol 520 & $005042.4+325459$ & 1.79 & M31 & 0.63 & -312 & 0.22 & 0.12 & -34 \\
\hline And XIV & $005135.0+294149$ & 2.04 & M31 & 0.73 & -481 & 0.18 & 0.16 & -211 \\
\hline And XIII & $005151.0+330016$ & 2.55 & M31 & 0.84 & -195 & 0.12 & 0.12 & 82 \\
\hline And IX & $005252.8+431200$ & 3.65 & M31 & 0.79 & -216 & 0.05 & 0.04 & 77 \\
\hline PAndAS-48 & $005928.2+312910$ & 2.31 & M31 & 0.82 & -250 & 0.15 & 0.14 & 19 \\
\hline And XVI & $005929.8+322236$ & 1.32 & M31 & 0.52 & -385 & 0.32 & 0.13 & -114 \\
\hline LGS 3 & $000355.0+215306$ & 1.37 & M31 & 0.65 & -286 & 0.30 & 0.27 & -44 \\
\hline IC 1613 & $000447.8+020800$ & 0.64 & M31 & 0.76 & -232 & 0.54 & 0.53 & -59 \\
\hline And $X$ & $000633.7+444816$ & 1.90 & M31 & 0.63 & -164 & 0.20 & 0.08 & 124 \\
\hline And V & $001007.1+473741$ & 2.64 & M31 & 0.81 & -403 & 0.11 & 0.11 & -113 \\
\hline And XV & $001418.7+380703$ & 2.64 & M31 & 0.76 & -323 & 0.11 & 0.09 & -49 \\
\hline And II & $001629.8+332509$ & 1.82 & M31 & 0.65 & -194 & 0.21 & 0.14 & 69 \\
\hline And XXIV & $001830.0+462158$ & 1.65 & M31 & 0.60 & -128 & 0.24 & 0.11 & 156 \\
\hline And XXII & $002740.0+280525$ & 1.75 & M31 & 0.79 & -127 & 0.23 & 0.22 & 116 \\
\hline And XXIII & $002921.8+384308$ & 2.24 & M31 & 0.73 & -243 & 0.15 & 0.13 & 23 \\
\hline M 33 & $003350.8+303937$ & 1.63 & M31 & 0.93 & -182 & 0.25 & 0.20 & 63 \\
\hline Perseus I & $030123.6+405918$ & 1.14 & M31 & 0.79 & -326 & 0.36 & 0.35 & -116 \\
\hline And XXVIII & $223241.2+311258$ & 1.04 & M31 & 0.65 & -331 & 0.39 & 0.38 & -3 \\
\hline Lac I & $225816.3+411728$ & 1.50 & M31 & 0.76 & -198 & 0.27 & 0.26 & 137 \\
\hline Cas dSph & $232631.8+504032$ & 1.73 & M31 & 0.82 & -307 & 0.23 & 0.22 & 24 \\
\hline Pegasus & $232834.1+144448$ & 0.73 & M31 & 0.97 & -184 & 0.50 & 0.42 & 89 \\
\hline Peg dSph & $235146.4+243510$ & 1.48 & M31 & 0.82 & -345 & 0.28 & 0.27 & -55 \\
\hline And XXI & $235447.7+422815$ & 2.42 & M31 & 0.86 & -361 & 0.14 & 0.12 & -43 \\
\hline And XXIX & $235855.6+304520$ & 1.87 & M31 & 0.73 & -194 & 0.21 & 0.19 & 106 \\
\hline PAndAS -04 & $000442.9+472142$ & 2.5 & M31 & 0.78 & -397 & & 0.12 & -79 \\
\hline PAndAS -05 & $000524.1+435535$ & 2.8 & M31 & 0.78 & -183 & $\ldots$ & 0.10 & 132 \\
\hline PAndAS -50 & $010150.6+481819$ & 2.7 & M31 & 0.78 & -323 & $\ldots$ & 0.11 & -29 \\
\hline PAndAS -56 & $012303.5+415511$ & 2.7 & M31 & 0.78 & -239 & $\ldots$ & 0.10 & 36 \\
\hline PAndAS -57 & $012747.5+404047$ & 2.6 & M31 & 0.78 & -186 & $\ldots$ & 0.12 & 84 \\
\hline PAndAS -58 & $012902.1+404708$ & 2.5 & M31 & 0.78 & -167 & $\ldots$ & 0.12 & 103 \\
\hline PAndAS -01 & $235712.0+433308$ & 2.6 & M31 & 0.78 & -333 & $\ldots$ & 0.12 & -15 \\
\hline PAndAS -02 & $235755.6+414649$ & 2.6 & M31 & 0.78 & -266 & $\ldots$ & 0.11 & 50 \\
\hline
\end{tabular}

M31 satellites, And XIV and And XII. Excluding these objects reduces the mass estimates by $14-15 \%$ in both cases.

\section{Hubble flow around the Local Group}

The proximate velocity field around the Local Group was considered in most detail by Karachentsev \& Kashibadze (2006) and Karachentsev et al. (2009). For a sample of 30 galaxies with TRGB distances from 0.7 to $3.0 \mathrm{Mpc}$ with respect to the Local
Group centre, it was shown that the Hubble flow is characterized by the local Hubble parameter $H_{\mathrm{loc}}=(78 \pm 2) \mathrm{km} \mathrm{s}^{-1} \mathrm{Mpc}^{-1}$, the radial velocity dispersion $\sigma_{v} \simeq 25 \mathrm{~km} \mathrm{~s}^{-1}$, and the radius of zero-velocity surface $R_{0}=(0.96 \pm 0.03) \mathrm{Mpc}$. The minimal value of $\sigma_{v}$ corresponded to the barycentre position of $D_{c}=(0.55 \pm 0.05) D_{\mathrm{M} 31}=0.43 \mathrm{Mpc}$, determining the mass ratio of $M_{\mathrm{MW}} / M_{\mathrm{M} 31} \simeq 0.8$ stated above.

In that approach, we considered the so-called minor attractor model, illustrated by the upper panel of Fig. 3. Here, a galaxy 
O. G. Kashibadze and I. D. Karachentsev: Cosmic flow around local massive galaxies

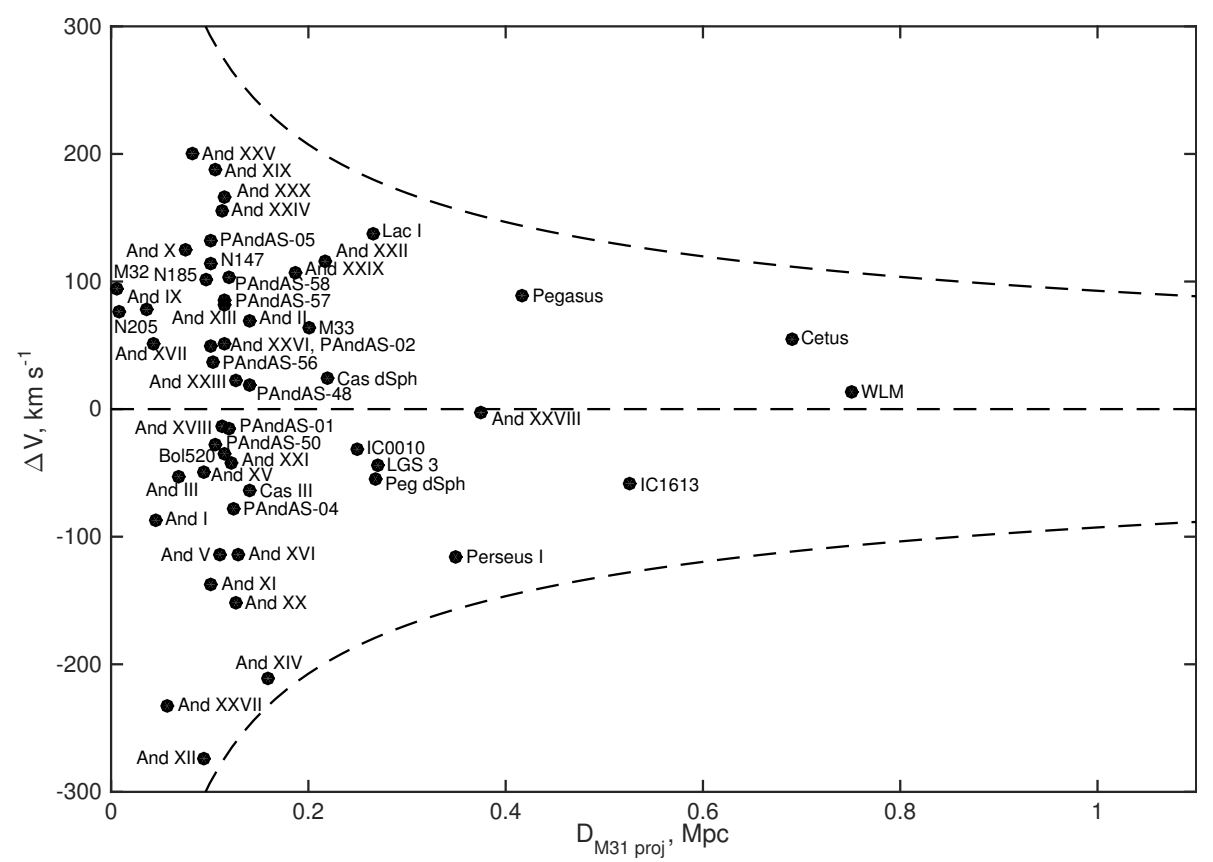

Fig. 2. Distribution of 52 test particles by their differential radial velocities and projected distances from M31. Dashed lines correspond to the parabolic velocity for a point mass of $1 \times 10^{12} M_{\odot}$.

Table 3. Total mass estimates for the Milky Way and M31 (in $10^{12} M_{\odot}$ ).

\begin{tabular}{|c|c|c|c|c|}
\hline $\mathrm{M}(\mathrm{MW})$ & $\mathrm{M}(\mathrm{M} 31)$ & $\mathrm{M}(\mathrm{MW}+\mathrm{M} 31)$ & $\mathrm{M}(\mathrm{M} 31) / \mathrm{M}(\mathrm{MW})$ & Reference \\
\hline $0.75 \pm 0.25$ & $\ldots$ & $\ldots$ & $\ldots$ & (1) \\
\hline$\ldots$ & $\ldots$ & $3.2 \pm 0.6$ & $\ldots$ & (2) \\
\hline$\ldots$ & $1.35 \pm 0.20$ & $\ldots$ & $\ldots$ & (3) \\
\hline $0.80 \pm 0.50$ & $1.70 \pm 0.30$ & $2.5 \pm 0.6$ & 2.3 & (4) \\
\hline $0.7 \pm 0.4$ & $\ldots$ & $\ldots$ & $\ldots$ & (5) \\
\hline $1.6 \pm 0.4$ & $1.8 \pm 0.5$ & $3.4 \pm 0.6$ & 1.1 & (6) \\
\hline $1.35 \pm 0.47$ & $1.76 \pm 0.33$ & $3.1 \pm 0.6$ & 1.3 & (7) \\
\hline $1.2 \pm 0.4$ & $0.9 \pm 0.3$ & $2.1 \pm 0.5$ & 0.75 & (8) \\
\hline $0.70 \pm 0.51$ & $1.39 \pm 0.26$ & $2.1 \pm 0.6$ & 2.0 & (9) \\
\hline ... & $\ldots$ & $2.6 \pm 0.4$ & $\ldots$ & (10) \\
\hline $1.30 \pm 0.30$ & $\ldots$ & $\ldots$ & $\ldots$ & (11) \\
\hline $1.02 \pm 0.76$ & $\ldots$ & $\ldots$ & $\ldots$ & (12) \\
\hline $1.55 \pm 0.35$ & $\ldots$ & $\ldots$ & $\ldots$ & (13) \\
\hline 2.84 & 1.65 & 4.5 & 0.58 & (14) \\
\hline $1.18 \pm 0.18$ & $1.69 \pm 0.25$ & $2.9 \pm 0.3$ & 1.4 & (15) \\
\hline 1.2 & 1.7 & 2.9 & 1.3 & median \\
\hline
\end{tabular}

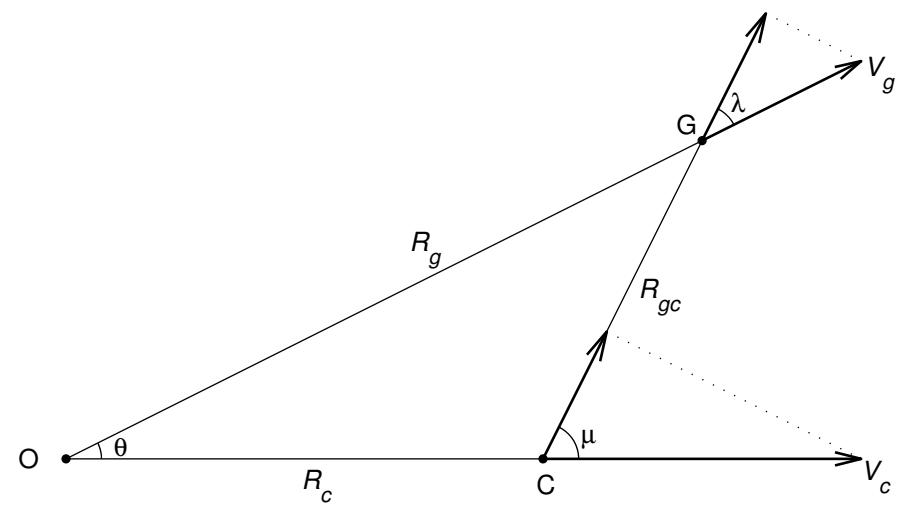

References. (1) Deason et al. (2012); (2) van der Marel et al. (2012); (3) Veljanoski et al. (2013); (4) Diaz et al. (2014); (5) Bhattacharjee et al. (2014); (6) Shull (2014); (7) Karachentsev \& Kudrya (2014); (8) Penarrubia et al. (2014); (9) Sofue (2015); (10) Penarrubia et al. (2016); (11) McMillan (2017); (12) Patel et al. (2017); (13) Fragione \& Loeb (2017); (14) Peebles (2017); (15) present paper.

group with centre, $C$, is separated by a distance, $D_{c}$, from the observer, $O$, and moves away along the line of site with the velocity, $V_{c}$. In the outskirts of the group there is a galaxy, $G$, with distance, $D_{g}$, and radial velocity, $V_{g}$. If the angle between $C$ and $G$ is $\theta$, then their mutual separation is expressed as

$R^{2}=D_{g}^{2}+D_{c}^{2}-2 D_{g} \times D_{c} \times \cos \theta$,

and the projected differential velocity is given by

$V_{g c}=V_{g} \times \cos \lambda-V_{c} \times \cos \mu$,

where $\mu=\lambda+\theta$, and

$\tan \lambda=D_{c} \times \sin \theta /\left(D_{g}-D_{c} \times \cos \theta\right)$.

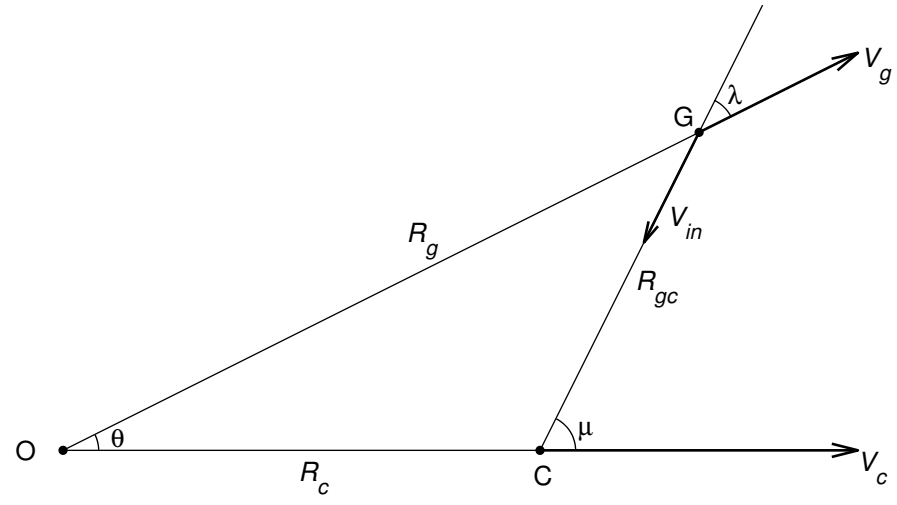

Fig. 3. Models of major and minor attractor. O represents an observer, $\mathrm{C}$ represents the centre of a galaxy group, and $\mathrm{G}$ represents a test particle (a galaxy).

In this scheme we assumed peculiar velocities of galaxies in the vicinity of a group to be small compared with velocities of the regular Hubble flow.

Yet, there is another possibility, which is the major attractor case (see lower panel of Fig. 3); this case is characterized by predominating infall towards the centre of a group or a cluster. 
Table 4. Isolated galaxies around the Local Group.

\begin{tabular}{|c|c|c|c|c|c|c|c|c|}
\hline Name & $\begin{array}{l}D_{\mathrm{MW}} \\
\mathrm{Mpc} \\
\end{array}$ & $\begin{array}{r}V_{\mathrm{h}} \\
\mathrm{km} \mathrm{s}^{-1} \\
\end{array}$ & $\begin{array}{c}R_{\mathrm{c}} \\
\mathrm{Mpc}\end{array}$ & $\begin{array}{r}V_{\mathrm{c}}^{\mathrm{mi}} \\
\mathrm{km} \mathrm{s}^{-1} \\
\end{array}$ & $\begin{array}{r}V_{\mathrm{c}}^{\mathrm{ma}} \\
\mathrm{km} \mathrm{s}^{-1}\end{array}$ & $T I$ & MD & $\begin{array}{r}\lambda \\
\operatorname{deg}\end{array}$ \\
\hline (1) & (2) & (3) & (4) & (5) & (6) & (7) & $(8)$ & $(9)$ \\
\hline WLM & 0.98 & -122 & 0.83 & -10 & -7 & -0.01 & M31 & 27 \\
\hline NGC 404 & 2.98 & -50 & 2.53 & 205 & 205 & -0.76 & Maffei2 & 1 \\
\hline KKs3 & 2.00 & 316 & 2.24 & 103 & 109 & -1.25 & MWay & 11 \\
\hline KKH 37 & 3.44 & 11 & 3.17 & 217 & 221 & -0.04 & M81 & 6 \\
\hline UGC 4879 & 1.37 & -25 & 1.34 & 25 & 33 & -0.63 & M31 & 19 \\
\hline Leo A & 0.74 & 24 & 0.93 & -47 & -53 & 0.03 & MWay & 29 \\
\hline Sex B & 1.43 & 300 & 1.71 & 94 & 101 & -0.82 & MWay & 13 \\
\hline NGC 3109 & 1.34 & 403 & 1.73 & 94 & 96 & -0.33 & Antlia & 9 \\
\hline Sex A & 1.45 & 324 & 1.78 & 78 & 82 & -0.83 & MWay & 11 \\
\hline Leo $\mathrm{P}$ & 1.73 & 262 & 1.95 & 120 & 128 & -1.07 & MWay & 12 \\
\hline NGC 3741 & 3.22 & 229 & 3.27 & 255 & 262 & -0.69 & M81 & 8 \\
\hline DDO 99 & 2.65 & 251 & 2.76 & 247 & 255 & -0.62 & NGC 4214 & 9 \\
\hline IC 3104 & 2.36 & 429 & 2.73 & 159 & 162 & -1.12 & NGC 4945 & 6 \\
\hline DDO 125 & 2.61 & 206 & 2.69 & 242 & 251 & -0.94 & M81 & 10 \\
\hline DDO 147 & 3.01 & 331 & 3.14 & 342 & 350 & -0.60 & NGC 4214 & 8 \\
\hline GR 8 & 2.19 & 217 & 2.48 & 128 & 132 & -1.37 & MWay & 9 \\
\hline UGC 8508 & 2.67 & 56 & 2.66 & 176 & 184 & -0.80 & M81 & 10 \\
\hline DDO 181 & 3.10 & 214 & 3.19 & 278 & 285 & -0.87 & NGC 4736 & 8 \\
\hline DDO 183 & 3.31 & 188 & 3.41 & 247 & 253 & -0.79 & NGC 4736 & 8 \\
\hline ККН 86 & 2.61 & 287 & 2.93 & 198 & 202 & -1.38 & NGC 5128 & 7 \\
\hline UGC 8833 & 3.25 & 221 & 3.37 & 273 & 280 & -0.89 & NGC 4736 & 8 \\
\hline KK 230 & 2.21 & 63 & 2.34 & 120 & 127 & -1.34 & M81 & 11 \\
\hline DDO 187 & 2.30 & 160 & 2.51 & 171 & 178 & -1.44 & MWay & 10 \\
\hline DDO 190 & 2.83 & 150 & 2.88 & 258 & 267 & -1.18 & M81 & 9 \\
\hline ESO 274-01 & 2.79 & 524 & 3.20 & 327 & 329 & -0.51 & NGC 5128 & 4 \\
\hline KKR 25 & 1.91 & -79 & 1.84 & 126 & 137 & -0.98 & M31 & 14 \\
\hline IC 4662 & 2.55 & 302 & 2.87 & 131 & 135 & -1.24 & NGC 5128 & 7 \\
\hline NGC 6789 & 3.55 & -140 & 3.28 & 153 & 156 & -1.32 & M81 & 6 \\
\hline Sag dIr & 1.08 & -79 & 1.19 & 20 & 29 & -0.44 & MWay & 22 \\
\hline DDO 210 & 0.98 & -140 & 0.98 & 12 & 22 & -0.31 & MWay & 27 \\
\hline IC 5152 & 1.96 & 122 & 2.08 & 70 & 77 & -1.20 & NGC 253 & 12 \\
\hline KK 258 & 2.24 & 92 & 2.18 & 151 & 160 & -0.91 & NGC 253 & 12 \\
\hline Tucana & 0.92 & 194 & 1.14 & 61 & 77 & -0.24 & MWay & 22 \\
\hline UGCA 438 & 2.22 & 62 & 2.15 & 101 & 108 & -0.48 & NGC 55 & 12 \\
\hline КKH 98 & 2.58 & -132 & 2.14 & 171 & 171 & -0.93 & M31 & 2 \\
\hline
\end{tabular}

If $V_{i}$ is the infall velocity than

$V_{g}=V_{c} \times \cos \theta-V_{i} \times \cos \lambda$,

and the velocity of a galaxy relative to the group centre is expressed as

$V_{i}=\left[V_{c} \times \cos \theta-V_{g}\right] / \cos \lambda$.

Evidently the difference between these two models would be insignificant if the galaxy lays almost strictly behind $(\lambda \simeq 0)$ or in front $\left(\lambda \simeq 180^{\circ}\right)$ of the group centre.

The last few years astronomers have detected some new dwarf galaxies in the vicinity of the Local Group (KKs3, LeoP, and KK258) and measured their accurate TRGB distances and radial velocities. For some galaxies (KKR25 and Tucana), old inexact values of radial velocities were corrected and distances were refined. This circumstance has motivated us to redefine parameters of the local Hubble flow.

To reduce the role of virial motions, we excluded galaxies with $T I>0$ from consideration; thus, the MW and M31 satellites with distances $D_{\mathrm{MW}}<0.8 \mathrm{Mpc}$ were consequently excluded. The data on the rest field galaxies with $D_{\mathrm{MW}}<$ $3.5 \mathrm{Mpc}$ are presented in Table 4 . The columns of Table 4 contain (1) galaxy name; (2) distance (in Mpc) from the Milky Way; (3) heliocentric radial velocity (in $\mathrm{km} \mathrm{s}^{-1}$ ); (4) distance from the
Local Group barycentre located at $D_{c}=0.43 \mathrm{Mpc} ;(5,6)$ velocity (in $\mathrm{km} \mathrm{s}^{-1}$ ) relative to the barycentre in the case of minor and major attractor, respectively; (7) tidal index; (8) the main disturber name; and (9) $\lambda$ in degrees (see Fig. 3).

The distribution of 35 isolated galaxies by distances and velocities relative to the Local Group barycentre for the case of minor attractor is presented in the upper panel of Fig. 4. As shown in the Table 4 data, only 14 galaxies of 35 have the MW or M31 as the main disturber; they are denoted by solid circles. With reference to these objects, the zone affected gravitationally by the Local Group reaches $R_{c} \simeq 2.5 \mathrm{Mpc}$, while more distant field galaxies are influenced by other massive neighbours of the Local Group, such as M81, NGC 253, and NGC 5128.

According to Peirani \& de Freitas Pacheco (2008), Falco et al. (2014), and Penarrubia \& Fattahi (2017), the radial velocity profile around the spherically symmetrical group or cluster can be expressed as

$V(R)=H_{0} \times R-H_{0} \times R_{0} \times\left(R_{0} / R\right)^{1 / 2}$,

where $R_{0}$ is the radius of the zero-velocity surface to be found. The solid line in Fig. 4 corresponds to Eq. (14) with parameters defined from the least squares method, $R_{0}=0.95 \pm 0.03 \mathrm{Mpc}$, $H_{0}=90 \pm 2 \mathrm{~km} \mathrm{~s}^{-1} \mathrm{Mpc}^{-1}$, and $\sigma_{v}=42 \mathrm{~km} \mathrm{~s}^{-1}$. The errors of $R_{0}$ and $H_{0}$ parameters were estimated using the Monte Carlo method, assuming that distance errors for galaxies are distributed 
O. G. Kashibadze and I. D. Karachentsev: Cosmic flow around local massive galaxies
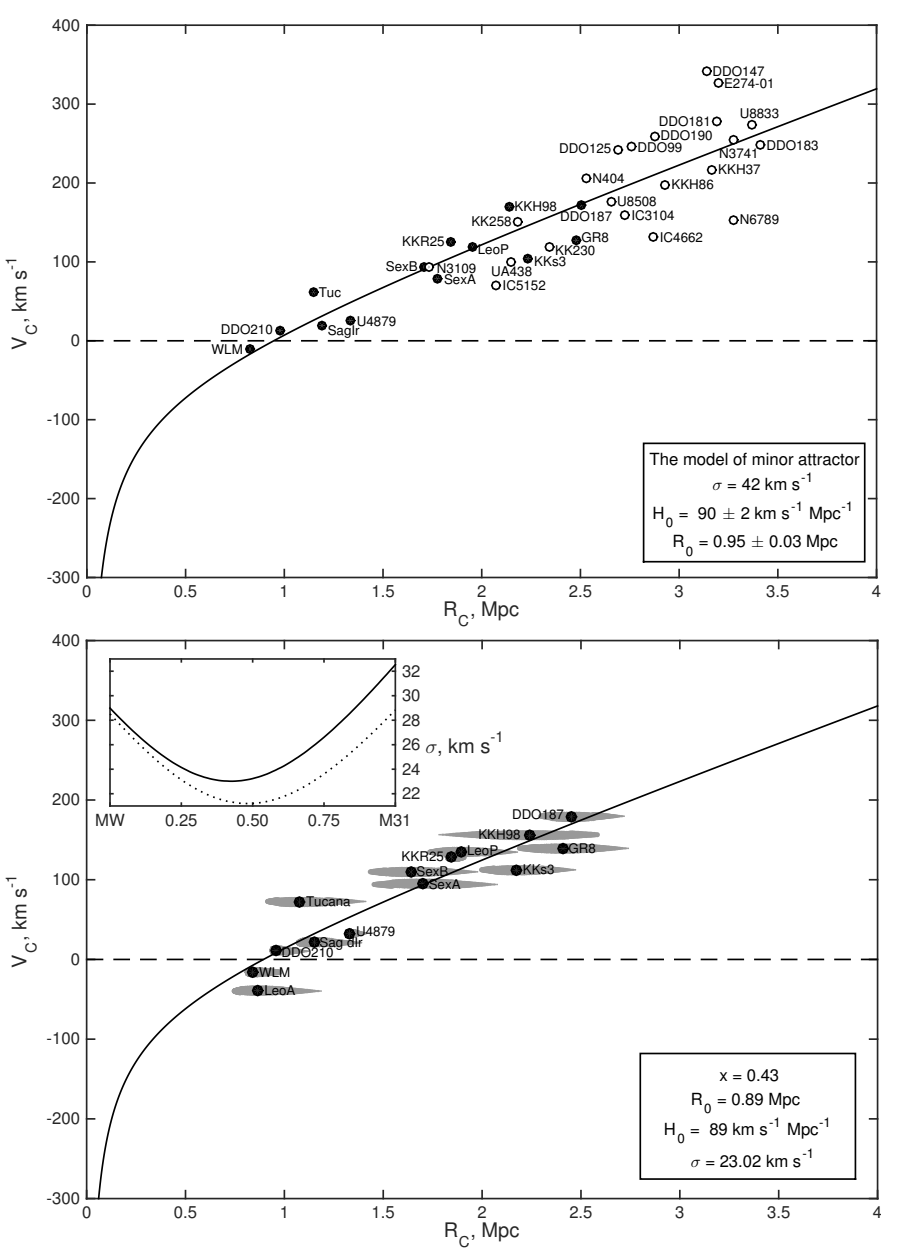

Fig. 4. Distribution of isolated galaxies by distances and velocities relative to the Local group barycentre assuming the minor attractor model. Upper panel: barycentre position locates at $x=D_{\mathrm{c}} / D_{\mathrm{M} 31}=0.55$ towards the M31. The solid circles indicate galaxies that have the MW or M31 as the main disturber. Lower panel: Hubble flow around the LG at the barycentre position of $x=D_{\mathrm{c}} / D_{\mathrm{M} 31}=0.43$. The grey wedges trace the companion positions under different $x$, where their thin end corresponds to the barycentre coinciding with M31. Solid and dotted lines in the insert indicate the velocity dispersion as a function of $x$ with and without the Leo A, respectively.

normally with a typical value of $\sim 5 \%$. The peculiar velocity dispersion in the upper panel of Fig. 4 is contributed mostly by distant galaxies, which are disturbed by the neighbouring groups. Considering the only 14 galaxies in the zone affected gravitationally by the Local Group, we obtain the following parameters for the surrounding Hubble flow: $R_{0}=0.85 \pm 0.03 \mathrm{Mpc}$, $H_{0}=79 \pm 3 \mathrm{~km} \mathrm{~s}^{-1} \mathrm{Mpc}^{-1}$, and $\sigma_{v}=23 \mathrm{~km} \mathrm{~s}^{-1}$. In the major attractor model, these parameters vary slightly, since $\lambda$ values for these 14 galaxies are small (see the last column in Table 4).

Three parameters, i.e. $R_{0}, H_{0}$, and $\sigma_{v}$, characterizing the local cosmic expansion, moderately depend on the position of the LG barycentre. Above, we used the barycentre location at the distance of $D_{c}=0.55 D_{\mathrm{M} 31}=0.43 \mathrm{Mpc}$, corresponding to the mass ratio of $M_{\mathrm{M} 31} / M_{\mathrm{MW}}=1.2$. This ratio matches well with the medians in Table 3. However, Penarrubia et al. (2014) found that the minimal scatter of nearby galaxies within $3 \mathrm{Mpc}$ around the LG is achieved with $M_{\mathrm{M} 31} / M_{\mathrm{MW}}=0.75$. The authors have concluded that their analysis rules out models in which M31 is more massive than our Galaxy with about 95\% confidence. To check this statement, we calculated $\sigma_{v}$ for 14 nearest
Table 5. Parameters of the local Hubble flow as a function of the LG barycentre position.

\begin{tabular}{llllllllll}
\hline$x=D_{\mathrm{C}} / D_{\mathrm{M} 31}$ & 0.10 & 0.20 & 0.30 & 0.40 & 0.50 & 0.60 & 0.70 & 0.80 & 0.90 \\
$M_{\mathrm{M} 31} / M_{\mathrm{MW}}$ & 0.11 & 0.25 & 0.43 & 0.67 & 1.00 & 1.50 & 2.33 & 4.00 & 9.00 \\
$\sigma_{v}, \mathrm{~km} \mathrm{~s}^{-1}$ & 26.7 & 24.9 & 23.6 & 23.1 & 23.4 & 24.2 & 25.7 & 27.7 & 30.0 \\
$R_{0}, \mathrm{Mpc}$ & 0.83 & 0.85 & 0.87 & 0.89 & 0.91 & 0.93 & 0.95 & 0.97 & 0.99 \\
\hline
\end{tabular}

isolated galaxies as a function of the position of the LG barycentre $x=D_{c} / D_{\mathrm{M} 31}$ on the line connecting the MW with M31. The data on $\sigma_{v}$ and $R_{0}$ are presented in Table 5. The lower panel of Fig. 4 shows the local Hubble diagram for 14 galaxies at various M31-to-MW mass ratios. Each galaxy is drawn by grey wedge with caliber inversely related to the dispersion $\sigma_{v}$ at given barycentre position; thus its thinner end indicates the barycentre position at M31. The insert in the figure shows the velocity scatter of galaxies respect to the best-fitting regression line. The solid and dotted lines in the insert represent the behaviour of $\sigma_{v}$ for a case of included or excluded Leo A, respectively. This dwarf galaxy is a marginally isolated object with the tidal index $T I=+0.03$. The derived minimums of these two lines fix the M31-to-MW mass ratio near 0.7 and 1.0, respectively, not allowing a firm assessment of which galaxy mass is dominated. Over the range of $M_{\mathrm{M} 31} / M_{\mathrm{MW}}=$ [1/3-3] the value of the zero velocity radius is changing within $R_{0}=0.86-0.96 \mathrm{Mpc}$. Thus, the observed coldness of the local Hubble flow leads us to measure the radius of the sphere separating the Local Group from the global cosmic expansion with $\sim 5 \%$ error. According to (4), the radius $R_{0}=0.91 \pm 0.05 \mathrm{Mpc}$ yields the total mass estimate for the Local Group $M_{T}=(1.5 \pm 0.2) \times 10^{12} M_{\odot}$ with an unprecedented accuracy, although this quantity lies below all values of $M(\mathrm{MW}+\mathrm{M} 31)$ in Table 3 . The mismatch becomes slightly less dramatic when the Planck model parameters in (4) replace the WMAP parameters as follows: $\Omega_{\mathrm{m}}=0.24, \Omega_{\lambda}=0.76$, and $H_{0}=$ $73 \mathrm{~km} \mathrm{~s}^{-1} \mathrm{Mpc}^{-1}$ (Spergel 2007); this increases the coefficient in (4) from 1.95 to 2.12 .

As noted by Chernin et al. (2004), the actual deviation of the binary shape of the Local Group from the spherical symmetry produces a minor bias in the $R_{0}$ and mass estimate. According to $N$-body simulations by Penarrubia et al. (2014), neglecting the quadrupole potential overestimates the Local Group mass up to $\sim 30 \%$.

\section{Other massive galaxies in the Local volume}

Considering the Hubble flow around other giant galaxies of the Local Volume, we selected 15 galaxies with stellar masses $M^{*}>3 \times 10^{10} M_{\odot}$ and accurate distances. Their overview is presented in Table 6 with objects ranging by their distances from the observer. For each of these 15 galaxies, surrounded by a suite of satellites, the second most massive member of its group is also indicated. In some cases, i.e. M31 and the Milky Way, NGC 5128 (CenA) and NGC 5236, Maffei 2, and IC 342, the second galaxy is comparable in mass with the first galaxy and acts itself as the centre of a dynamically separated subgroup.

The columns of Table 6 contains (1) galaxy name; $(2,3)$ its supergalactic coordinates; (4) the galaxy distance from the MW; (5) its radial velocity relative to the Local Group centroid; (6) logarithmic stellar mass; (7) logarithmic orbital mass according to Karachentsev \& Kudrya (2014); (8) number of satellites of the main galaxy with measured radial velocities and accurate distances. 
Table 6. Giant galaxies in the Local Volume.

\begin{tabular}{|c|c|c|c|c|c|c|c|}
\hline Galaxy & $\begin{array}{r}\text { SGL } \\
\text { deg }\end{array}$ & $\begin{array}{r}\text { SGB } \\
\text { deg } \\
\end{array}$ & $\begin{array}{l}D_{\mathrm{MW}} \\
\mathrm{Mpc}\end{array}$ & $\begin{array}{r}V_{\mathrm{LG}} \\
\mathrm{km} \mathrm{s}^{-1} \\
\end{array}$ & $\begin{array}{r}\lg M^{*} \\
M_{\odot}\end{array}$ & $\begin{array}{c}\lg M_{\text {orb }} \\
M_{\odot}\end{array}$ & $\begin{array}{c}N_{\text {sat }} \\
(V, D)\end{array}$ \\
\hline (1) & (2) & (3) & (4) & (5) & (6) & $(7)$ & $(8)$ \\
\hline M31 & 336.19 & 12.55 & 0.78 & -29 & 10.79 & 12.49 & 90 \\
\hline M Way & $\ldots$ & $\ldots$ & 0.01 & -65 & 10.70 & & \\
\hline M81 & 41.12 & 0.59 & 3.70 & 104 & 10.95 & 12.69 & 22 \\
\hline M 82 & 40.72 & 1.05 & 3.61 & 328 & 10.59 & & \\
\hline NGC 5128 & 159.75 & -5.25 & 3.68 & 310 & 10.89 & 12.89 & 28 \\
\hline NGC 5236 & 147.93 & 0.99 & 4.90 & 307 & 10.86 & & \\
\hline Maffei 2 & 359.58 & 0.83 & 3.48 & 214 & 10.86 & 12.51 & 3 \\
\hline IC 342 & 10.60 & 0.37 & 3.28 & 244 & 10.60 & & \\
\hline NGC 253 & 271.57 & -5.01 & 3.70 & 276 & 10.98 & 12.18 & 7 \\
\hline NGC 247 & 275.92 & -3.73 & 3.72 & 216 & 9.50 & & \\
\hline NGC 4826 & 95.61 & 6.13 & 4.41 & 365 & 10.49 & 10.78 & 4 \\
\hline DDO 154 & 90.13 & 6.90 & 4.04 & 354 & 7.59 & & \\
\hline NGC 4736 & 76.24 & 9.50 & 4.41 & 352 & 10.56 & 12.43 & 16 \\
\hline NGC 4449 & 72.30 & 6.18 & 4.27 & 249 & 9.68 & & \\
\hline M 101 & 63.58 & 22.61 & 6.95 & 378 & 10.79 & 12.17 & 6 \\
\hline NGC 5474 & 64.30 & 22.93 & 6.98 & 424 & 9.21 & & \\
\hline NGC 4258 & 68.74 & 5.55 & 7.66 & 506 & 10.92 & 12.50 & 7 \\
\hline NGC 4242 & 70.28 & 4.81 & 7.9: & 568 & 9.47 & & \\
\hline NGC 5055 & 76.20 & 14.25 & 9.04 & 562 & 11.00 & 12.49 & 0 \\
\hline NGC 4460 & 71.58 & 6.48 & 9.59 & 551 & 9.66 & & \\
\hline NGC 4594 & 126.69 & -6.68 & 9.30 & 894 & 11.30 & 13.45 & 0 \\
\hline NGC 4597 & 121.05 & -5.12 & 10.1: & 912 & 9.48 & & \\
\hline NGC 6744 & 208.10 & 10.38 & 9.51 & 706 & 10.91 & 11.72 & 4 \\
\hline NGC 6684 & 205.81 & 9.11 & 8.7: & 720 & 10.39 & & \\
\hline NGC 3115 & 112.40 & -42.86 & 9.68 & 439 & 10.95 & 12.54 & 0 \\
\hline P 4078671 & 114.10 & -45.34 & 9.38 & 378 & 7.95 & & \\
\hline NGC 2683 & 55.87 & -33.42 & 9.82 & 334 & 10.81 & 12.13 & 2 \\
\hline KK 69 & 55.64 & -33.09 & 9.16 & 418 & 7.27 & & \\
\hline NGC 3379 & 93.64 & -25.85 & 11.32 & 774 & 10.92 & 13.23 & 3 \\
\hline NGC 3368 & 94.29 & -26.41 & 10.42 & 740 & 10.83 & & \\
\hline
\end{tabular}

Aside from the galaxies presented in Table 6, the Local Volume contains another two massive galaxies - NGC 2903 $\left(\log M^{*}=10.82\right)$ and NGC $6946\left(\log M^{*}=10.76\right)$. But their distances measured from the luminosity of brightest stars are not yet sufficiently accurate. In total, the 15 giant galaxies have about 500 satellites in their suites, but, as shown in the last column of Table 6, only 102 satellites outside the Local Group have accurate estimates of distances and velocities. Among the second most massive members of 15 groups, three galaxies - NGC 4242, NGC 4597 and NGC 6684 - have Tully-Fisher distances with accuracy of $\sim 20 \%$ (denoted with column signs). In 11 of 15 groups, the main galaxy exceeds its satellites twice or more in mass, allowing us to estimate its halo mass from the orbital motions. This approach is not worthwhile in the case of the rich group Leo I, where NGC 3379, NGC 3368, and several other bright members have compatible luminosities.

Despite the great efforts to measure highly accurate TRGB distances of nearby galaxies from Hubble Space Telescope data, many neighbouring groups stay still poorly explored. For example, in the outskirts of giant galaxies NGC 4594 (Sombrero),
NGC 5055, and NGC 3115, no satellites have reliable distance estimates.

\section{Cosmic flow around the synthetic (stacked) nearby group}

Seeking to use as much information as possible about companion motions around the nearby massive galaxies outside their virial zones, we combined the data on companions of various galaxies into the single synthetic group. To be included into the consolidated group, a galaxy should satisfy the following four conditions: (1) a companion has accurate estimates of distance and radial velocities; (2) the companion distance from the main galaxy, $R_{\mathrm{MG}}$, is less than $3.5 \mathrm{Mpc}$; (3) the companion belongs to field galaxies, having $T I<0$; and (4) the companion has a proper aspect, when its position angle $\lambda$ between the vector of companion radial velocity and the line joining it with the main galaxy (see Fig. 3) lays within $\lambda<45^{\circ}$ or $\lambda>135^{\circ}$.

These conditions are satisfied for 66 galaxies of the Local Volume; the corresponding data are presented in Table 7. Its 
O. G. Kashibadze and I. D. Karachentsev: Cosmic flow around local massive galaxies

Table 7. Isolated galaxies around the nearby group centres.

\begin{tabular}{|c|c|c|c|c|c|c|c|}
\hline Main gal. & Name & $\begin{array}{c}R_{\mathrm{C}} \\
\mathrm{Mpc}\end{array}$ & $\begin{array}{r}V_{\mathrm{mi}} \\
\mathrm{km} \mathrm{s}^{-1}\end{array}$ & $\begin{array}{r}V_{\mathrm{ma}} \\
\mathrm{km} \mathrm{s}^{-1}\end{array}$ & TI & MD & $\begin{array}{r}\lambda \\
\operatorname{deg}\end{array}$ \\
\hline (1) & (2) & (3) & (4) & (5) & (6) & (7) & (8) \\
\hline M81 & UGC 04879 & 2.40 & 141 & 147 & -0.63 & M31 & 153 \\
\hline M81 & UGC 06456 & 1.26 & -38 & -86 & -0.31 & M81 & 35 \\
\hline M81 & NGC 3738 & 2.16 & 150 & 170 & -1.01 & M81 & 33 \\
\hline M81 & UGC 06757 & 1.37 & 51 & 42 & -0.41 & M81 & 40 \\
\hline M81 & UGC 07242 & 2.06 & 56 & 48 & -0.40 & N4605 & 24 \\
\hline M81 & NGC 4236 & 1.13 & 17 & -15 & -0.16 & M81 & 44 \\
\hline M81 & NGC 4605 & 2.36 & 131 & 138 & -1.07 & M101 & 29 \\
\hline M81 & DDO165 & 1.71 & 56 & 41 & -0.64 & N4236 & 39 \\
\hline M81 & UGC 08245 & 1.54 & 14 & -27 & -0.58 & M81 & 40 \\
\hline NGC 5128 & NGC 3621 & 3.50 & 219 & 204 & -1.68 & N4594 & 34 \\
\hline NGC 5128 & ESO 320-014 & 2.66 & 134 & 100 & -0.68 & N3621 & 38 \\
\hline NGC 5128 & ESO 379-007 & 2.07 & 129 & 111 & -1.04 & N5236 & 45 \\
\hline NGC 5128 & ESO 381-018 & 1.45 & 81 & 74 & -0.40 & N5236 & 29 \\
\hline NGC 5128 & ESO 381-020 & 1.47 & 55 & 39 & -0.33 & N5236 & 29 \\
\hline NGC 5128 & ESO 443-009 & 1.97 & 124 & 119 & -0.53 & N5236 & 24 \\
\hline NGC 5128 & KK182 & 1.80 & 66 & 62 & -0.67 & N5236 & 16 \\
\hline NGC 5128 & ESO 270-017 & 2.86 & 282 & 289 & -1.35 & N5236 & 15 \\
\hline NGC 5128 & HIPASS J1348-37 & 1.45 & 56 & 54 & -0.21 & N5236 & 12 \\
\hline NGC 5128 & HIPASS J1351-47 & 1.80 & 20 & -11 & -0.87 & N5236 & 29 \\
\hline NGC 5128 & NGC 5408 & 1.28 & 0 & -25 & -0.35 & N5236 & 29 \\
\hline NGC 5128 & ESO 223-009 & 2.78 & 142 & 120 & -1.42 & N5236 & 33 \\
\hline Maffei2 & UGC 01281 & 3.00 & 215 & 223 & -1.20 & N784 & 37 \\
\hline Maffei2 & KK17 & 2.97 & 220 & 238 & -0.96 & N784 & 41 \\
\hline Maffei2 & NGC 0784 & 3.25 & 240 & 254 & -1.30 & U1281 & 37 \\
\hline Maffei2 & KKH18 & 2.45 & 207 & 243 & -1.17 & Maffei2 & 42 \\
\hline Maffei2 & KKH34 & 1.80 & 110 & 106 & -0.65 & M81 & 39 \\
\hline $\mathrm{N} 253$ & WLM & 2.76 & 288 & 298 & -0.01 & M31 & 161 \\
\hline $\mathrm{N} 253$ & NGC 0045 & 3.02 & 259 & 260 & -1.05 & N24 & 10 \\
\hline $\mathrm{N} 253$ & PiscesA & 3.49 & 278 & 292 & -1.68 & $\mathrm{~N} 253$ & 40 \\
\hline $\mathrm{N} 253$ & NGC 0059 & 1.35 & 163 & 174 & -0.37 & $\mathrm{~N} 253$ & 23 \\
\hline $\mathrm{N} 253$ & DDO226 & 1.24 & 136 & 138 & -0.27 & $\mathrm{~N} 253$ & 9 \\
\hline $\mathrm{N} 253$ & UGCA438 & 1.76 & 178 & 221 & -0.48 & N55 & 136 \\
\hline N4826 & AGC 749241 & 1.32 & 65 & 59 & -0.73 & N4656 & 20 \\
\hline N4826 & GR8 & 2.26 & 227 & 230 & -1.37 & MW & 165 \\
\hline N4826 & DDO187 & 2.34 & 202 & 207 & -1.44 & MW & 144 \\
\hline N4736 & NGC 3741 & 1.47 & 105 & 95 & -0.69 & M81 & 135 \\
\hline N4736 & DDO099 & 1.87 & 99 & 86 & -0.62 & N4214 & 153 \\
\hline N4736 & UGCA281 & 1.48 & 30 & 12 & -0.92 & N4258 & 24 \\
\hline N4736 & DDO126 & 0.76 & -64 & -136 & -0.02 & N4736 & 37 \\
\hline N4736 & DDO125 & 1.80 & 92 & 90 & -0.94 & M81 & 169 \\
\hline N4736 & Arp 211 & 1.78 & 146 & 146 & -0.86 & N4258 & 9 \\
\hline N4736 & DDO147 & 1.42 & -4 & -13 & -0.60 & N4214 & 164 \\
\hline N4736 & NGC 5023 & 1.72 & 141 & 141 & -0.89 & M101 & 13 \\
\hline N4736 & UGC 08508 & 1.94 & 172 & 183 & -0.80 & M81 & 144 \\
\hline N4736 & DDO181 & 1.44 & 73 & 59 & -0.87 & N4736 & 149 \\
\hline N4736 & DDO183 & 1.36 & 107 & 109 & -0.79 & N4736 & 136 \\
\hline N4736 & KK230 & 2.36 & 220 & 235 & -1.34 & M81 & 148 \\
\hline N4736 & DDO190 & 1.91 & 116 & 85 & -1.18 & M81 & 135 \\
\hline M101 & LV J1157+5638 & 3.00 & 190 & 215 & -0.80 & $\mathrm{~N} 4258$ & 45 \\
\hline M101 & NGC 4068 & 3.07 & 131 & 98 & -0.48 & N4736 & 137 \\
\hline M101 & $\mathrm{MCG}+09-20-131$ & 2.82 & 161 & 167 & -0.43 & N4736 & 137 \\
\hline M101 & UGC 07298 & 3.14 & 149 & 136 & -0.35 & N4736 & 142 \\
\hline M101 & NGC 4736 & 3.07 & 87 & 12 & -0.13 & N4449 & 136 \\
\hline M101 & NGC 5204 & 2.44 & 49 & 40 & -0.88 & N4736 & 162 \\
\hline M101 & NGC 5238 & 2.49 & 43 & 37 & -0.41 & N4736 & 166 \\
\hline M101 & KKH87 & 1.97 & 97 & 96 & -0.81 & N5194 & 11 \\
\hline M101 & DDO194 & 1.29 & 15 & -4 & -0.10 & N5585 & 150 \\
\hline N4258 & KK109 & 3.23 & 270 & 275 & -0.32 & N4736 & 164 \\
\hline N4258 & $\mathrm{MCG}+06-27-017$ & 3.05 & 185 & 180 & -0.22 & N4395 & 151 \\
\hline N4258 & NGC 4395 & 3.25 & 222 & 225 & -0.12 & N4736 & 146 \\
\hline N4258 & NGC 4707 & 1.38 & -9 & -66 & -0.45 & N4258 & 143 \\
\hline N4258 & NGC 4861 & 3.17 & 371 & 464 & -0.57 & N5055 & 37 \\
\hline N6744 & IC 4710 & 2.06 & 139 & 137 & -0.99 & N6684 & 158 \\
\hline N6744 & IC 4870 & 1.00 & 4 & -40 & -0.22 & N6744 & 142 \\
\hline N2683 & AGC182595 & 1.61 & 27 & 12 & -0.78 & N2683 & 144 \\
\hline N3379 & DDO088 & 3.20 & 328 & 329 & -0.45 & N3627 & 174 \\
\hline
\end{tabular}


Table 8. Parameters of the Hubble flow around the nearby synthetic group under various assumptions.

\begin{tabular}{rccc|ccc}
\hline \hline \multicolumn{1}{c}{ Case } & \multicolumn{3}{c}{ Minor attractor } & \multicolumn{3}{c}{ Major attractor } \\
\hline & $\begin{array}{c}H_{0} \\
\mathrm{~km} \mathrm{~s}^{-1} \mathrm{Mpc}^{-1}\end{array}$ & $\begin{array}{c}\sigma_{v} \\
\mathrm{~km} \mathrm{~s}^{-1}\end{array}$ & $\begin{array}{c}R_{0} \\
\mathrm{Mpc}\end{array}$ & $\begin{array}{c}H_{0} \\
\mathrm{~km} \mathrm{~s}^{-1} \mathrm{Mpc}^{-1}\end{array}$ & $\begin{array}{c}\sigma_{v} \\
\mathrm{~km} \mathrm{~s}^{-1}\end{array}$ & $\begin{array}{c}R_{0} \\
\mathrm{Mpc}\end{array}$ \\
\hline$(1)$ & $(2)$ & $(3)$ & $(4)$ & $(5)$ & $(6)$ & $(7)$ \\
\hline Main galaxy (MG) & 76 & 62 & 0.83 & 80 & 84 & 0.93 \\
MG-normalized & 80 & 65 & 0.71 & 82 & 89 & 0.76 \\
Barycentre (BC) & 85 & 57 & 0.93 & 85 & 84 & 1.03 \\
BC-normalized & 88 & 66 & 0.76 & 95 & 85 & 0.93 \\
\hline
\end{tabular}
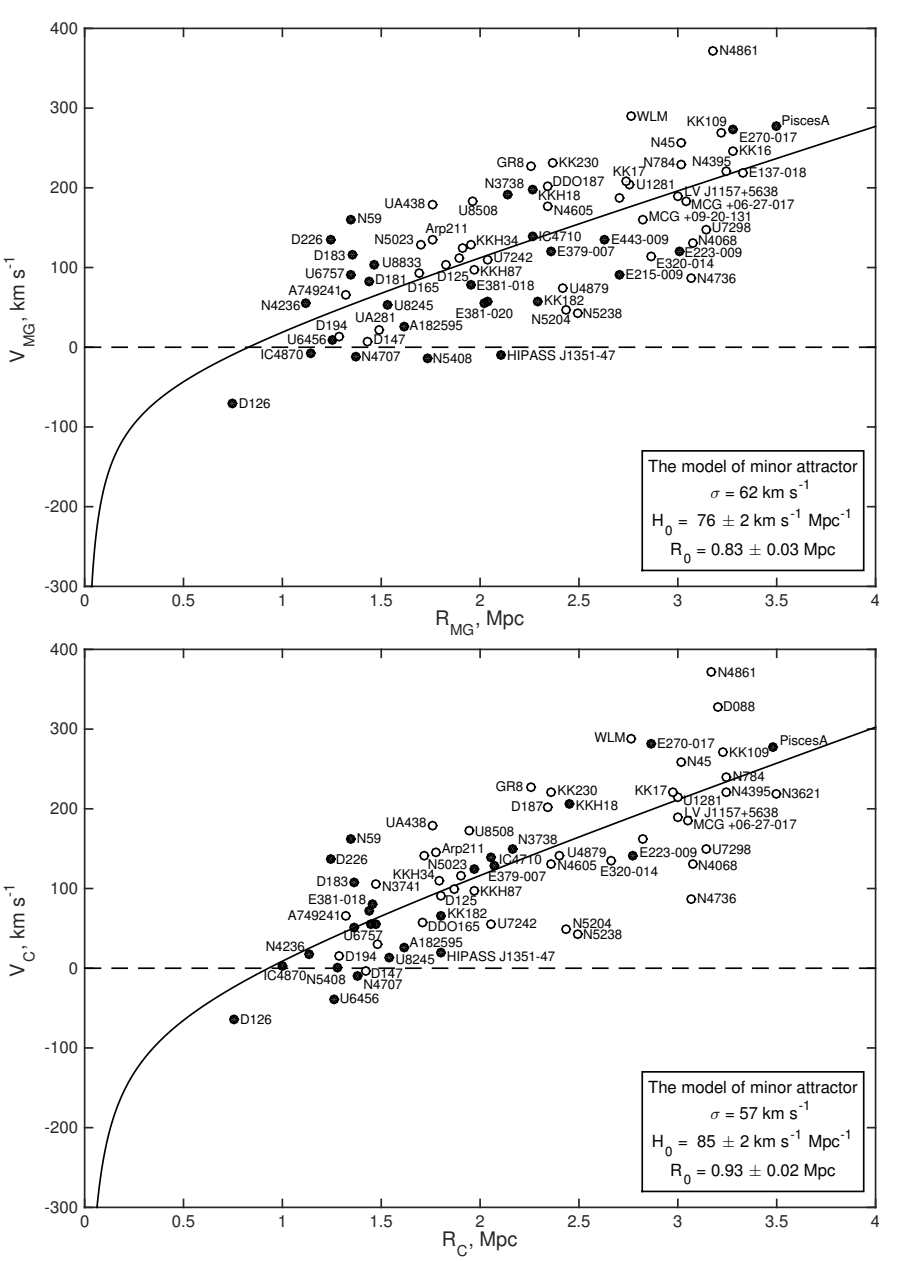

Fig. 5. Hubble diagram for the synthetic group of the Local Volume, assuming the minor attractor model. Upper panel: distances and velocities of satellites are calculated relative to the main galaxy in a group. Lower panel: distances and velocities of satellites are calculated relative to the barycentre of a pair of the most massive galaxies in each group.

columns contain: (1) name of the main galaxy acting as the centre of its suite; (2) name of a companion galaxy; (3) companion galaxy distance from the group barycentre; $(4,5)$ companion galaxy velocity relative to the group barycentre in the case of minor or major attractor; $(6,7)$ tidal index of the galaxy and the name of its main disturber; and (8) position angle of the companion as indicated in Fig. 3.

The Hubble diagram for the synthetic group of the Local Volume for the minor attractor model with distances and velocities calculated relative to the main galaxy is shown in the upper panel of Fig. 5. The cosmic flow around the synthetic group is characterized by the Hubble parameter $H_{0}=(76 \pm 2) \mathrm{km} \mathrm{s}^{-1} / \mathrm{Mpc}$, velocity dispersion $\sigma_{v}=62 \mathrm{~km} \mathrm{~s}^{-1}$, and radius of the zero velocity surface $R_{0}=0.83 \pm 0.03 \mathrm{Mpc}$. As one can see, the radius $R_{0}$ turned out to be quite small, corresponding to the effective mass of the synthetic group of $\sim 1.1 \times 10^{12} M_{\odot}$. To estimate how various factors influence $R_{0}$, we constructed another series of Hubble diagrams. An alternative Hubble diagram with distances and velocities calculated relative to the group barycentre rather than from the main galaxy itself is presented in the lower panel of Fig. 5. The barycentre is supposed to lie between the two most massive galaxies of each group given in Table 6. In this case the local Hubble parameter is $H_{0}=(85 \pm 2) \mathrm{km} \mathrm{s}^{-1} \mathrm{Mpc}$, peculiar velocity dispersion $\sigma_{v}=57 \mathrm{~km} \mathrm{~s}^{-1}$, and radius $R_{0}$ reaches $R_{0}=0.93 \pm 0.02 \mathrm{Mpc}$.

As follows from the data on Table 6, the nearby galaxy groups differ substantially in their stellar and virial masses, $M^{*}$ and $M_{\text {orb }}$, which can lead to a systematic bias in the averaged $R_{0}$ estimate. To verify this effect, we normalized distances of companions around each group to its individual radius $R_{0}$, assuming $R_{0} \propto M_{*}^{1 / 3}$ or $R_{0} \propto M_{\text {orb }}^{1 / 3}$. After that we did not find any decrease in peculiar velocity dispersion in the synthetic Hubble diagram.

The resulting values of $H_{0}, \sigma_{v}$, and $R_{0}$ parameters for all discussed cases are presented in Table 8; i.e. distances and velocities calculated relative to the main galaxy or group barycentre and the minor or major attractor model. These data allow us to conclude, first, that changing a model from a minor attractor to major attractor increases the $R_{0}$ estimate and causes a significant increment in dispersion, and, second, that accounting for the second most massive galaxy in a group leads to a notable growth of the $R_{0}$ estimate.

\section{Discussion}

As our estimates suggest, galaxies in the infall zone between the virial radius and the $R_{0}$ are relatively small in number, $\sim 15 \%$. This circumstance, inherent for the Local Group and for other nearby groups, opts for estimating $R_{0}$ value within minor attractor model. The low value of peculiar velocity dispersion resulting in this case is also an oblique argument for such a choice.

The second most massive galaxy plays an essential role in the kinematics of several nearby groups, often forming a dynamical subsystem. So, deciding on a barycentre of the two most bright galaxies as the reference point for distances and velocities of companions seems to be more preferable than the main galaxy itself. Hence, we adopt the value of $0.93 \pm 0.02 \mathrm{Mpc}$ as the optimal estimate for $R_{0}$ radius of the cumulative group (see the lower panel of Fig. 5). The corresponding mass is $\log \left(M_{T} / M_{\odot}\right)=12.20$ with a formal error of $\sim 0.04$ dex. Averaging orbital mass estimates from Table 6 and considering the representation of each group in the Hubble diagram, we obtain the mean logarithmic mass $\log \left(M_{\text {orb }} / M_{\odot}\right)=12.42 \pm 0.07$. So, the mass of the synthetic group derived from outer motions of surrounding galaxies turned out to be $\sim 60 \%$ of the expected 


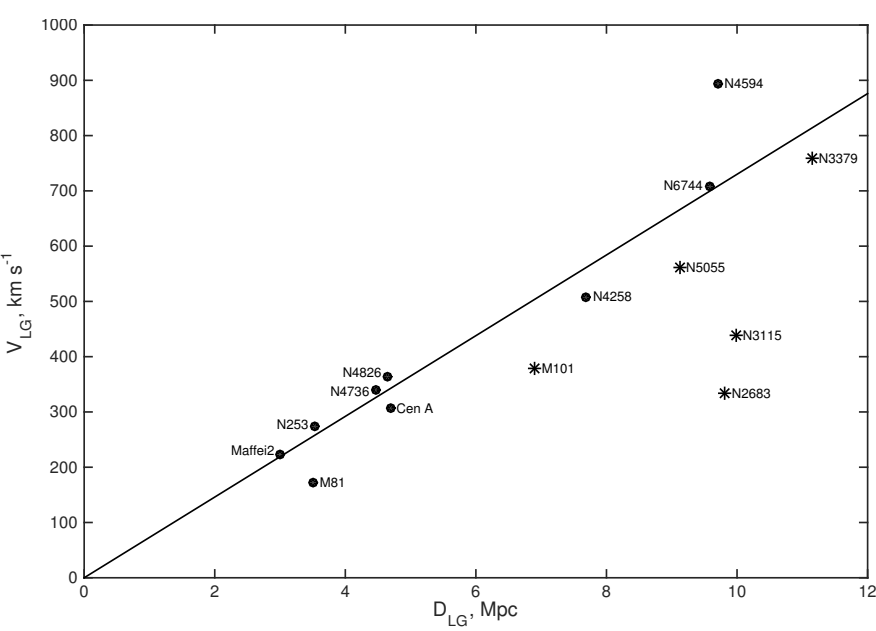

Fig. 6. Distribution of barycentres of 15 nearby groups by their radial velocities and distances from the Local Group centre. The solid line corresponds to the regular Hubble flow with Hubble parameter $H_{0}=$ $73 \mathrm{~km} \mathrm{~s}^{-1} \mathrm{Mpc}^{-1}$.

mass from inner orbital motions of satellites. A probable source of this discrepancy was discussed by Chernin et al. (2013) and Karachentsev \& Kudrya (2014).

As noted by Chernin et al. (2013), the estimate of the total mass of a group includes two components, $M_{T}=M_{\mathrm{m}}+M_{\mathrm{DE}}$, where $M_{\mathrm{m}}$ is the mass of dark and baryonic matter and $M_{\mathrm{DE}}$ is the mass, negative in magnitude, which is determined by the dark energy with the density of $\rho_{\mathrm{DE}}$,

$M_{\mathrm{DE}}=(8 \pi / 3) \times \rho_{\mathrm{DE}} \times R^{3}$.

On the scale of virial radius, the contribution of this component in the group mass does not exceed $1 \%$, but in the sphere of $R_{0}$ radius, the role of this kind of a mass defect becomes significant. In the standard $\Lambda$ CDM model with $\Omega_{\mathrm{m}}=0.24$ and $H_{0}=73 \mathrm{~km} \mathrm{~s}^{-1} \mathrm{Mpc}^{-1}$ the contribution of dark energy is

$\left(M_{\mathrm{DE}} / M_{\odot}\right)=-0.85 \times 10^{12} \times\left(R_{0} / \mathrm{Mpc}\right)^{3}$,

i.e. about $30 \%$ of the Local Group mass determined by orbital motions. This correction essentially reduces the observed discrepancy between the mass estimates for the Local Group, as well as for other nearby groups, derived via internal (virial) and external galaxy motions.

Another possible explanation might be caused by the existence of unrelaxed (tidal) thin planar structures of satellites seen around the MW and M31 (Kroupa 2014), which are at variance with the assumption of spherical symmetry case.

The peculiar velocity dispersion in the vicinity of the synthetic group, $57 \mathrm{~km} \mathrm{~s}^{-1}$, is twice as large as in the outskirts of the Local Group. This difference might originate from bulk motions of galaxies, which become perceptible on the scale of $\sim 5-10 \mathrm{Mpc}$. A giant galaxy is not necessarily the main disturber for neighbouring field galaxies. Indeed, this is the case for only a portion of companion objects presented in Table 7. Another portion, which are comprised of mostly distant field galaxies (shown by open circles), are gravitationally influenced by a massive galaxy from another neighbouring group.

Figure 6 reproduces the distribution of barycentres of 15 nearby groups listed in Table 5 by distances and radial velocities relative to the Local Group centre. The straight line corresponds to the regular Hubble flow with $H_{0}=$ $73 \mathrm{~km} \mathrm{~s}^{-1} \mathrm{Mpc}^{-1}$. Barycentres of the groups with $D_{\mathrm{LG}}<6 \mathrm{Mpc}$ situated in the supergalactic plane (i.e. in the Local Sheet; Tully et al. 2016) demonstrate a small scatter of radial velocities. More distant groups, around M 101, NGC 5055, NGC 2683, NGC 3115, and NGC 3379 at supergalactic latitudes $|S G B|>10^{\circ}$ (denoted with asterisks), exhibit negative peculiar velocities about $-200 \mathrm{~km} \mathrm{~s}^{-1}$. These velocities are caused by the observed expansion of the Local Void with an amplitude of $\sim 260 \mathrm{~km} \mathrm{~s}^{-1}$ (Tully et al. 2016). Also, the group around Sombrero (NGC 4594) is located just near the zero-velocity surface of the Virgo cluster. Its positive peculiar velocity reflects the group fall towards the cluster. Apparently, some portion of these bulk motions manifest themselves as extra peculiar velocities of the Local Volume galaxies in the panels of Fig. 5. Ignoring these non-virial coherent motions may lead to the overestimation of galaxy masses based on the Numerical Action Method (Peebles 2017).

Our conclusion that the peripheral regions of the Local Group and other neighbouring groups do not contain a large amount of dark matter seems to be the most important result of this work. The bulk of mass is concentrated within the virial radius of these groups. The same inference was made for the nearest Virgo cluster (Karachentsev et al. 2014) from the observed infall of galaxies towards the cluster centre. Yet further evidence is provided by Kourkchi \& Tully (2017), who have considered infall zones and collapsed cores of halos in the Local Universe.

A review of available observational data on distances and radial velocities of the Local Volume galaxies shows that the population of outskirts of the nearby groups has not yet been covered with highly accurate distance measurements. There are groups, for example around the giant Sombrero galaxy, totally lack reliable distance estimates, even for close probable satellites. The systematical measurements of TRGB distances with the Hubble Space Telescope within the Local Volume have the potential to provide meaningful data on the distribution of the dark matter on the scales of $\sim 1 \mathrm{Mpc}$.

Acknowledgements. The authors are grateful to Elena Kaisina for updating the Local Volume Galaxies Database (https://www . sao.ru/lv/lvgdb/), Dmitry Makarov for the renewed data on galaxy tidal indices, and Brent Tully for reviewing the manuscript and for his helpful comments. This work is supported by the Russian Science Foundation grant No. 14-12-00965.

\section{References}

Abazajian, K. N., Adelman-McCarthy, J. K., Agüeros, M. A., et al. 2009, ApJS, 182,543

Barber, C., Starkenburg, E., Navarro, J. F., et al. 2014, MNRAS, 437, 959

Bhattacharjee, P., Chaudhury, S., \& Kundu, S. 2014, ApJ, 785, 63

Chernin, A. D., Karachentsev, I. D., Valtonen, M. J., et al. 2004, A\&A, 415, 19

Chernin, A. D., Bisnovatyi-Kogan, G. S., Teerikorpi, P., et al. 2013, A\&A, 553, A101

Deason, A. J., Belokurov, V., Evans, N. W., et al. 2012, MNRAS, 425, 2840

de Vaucouleurs, G. 1958, AJ, 63, 253

de Vaucouleurs, G. 1964, AJ, 69, 737

de Vaucouleurs, G. 1972, in External Galaxies and Quasi-Stellar Objects, eds.

D. S. Evans, D. Wills, \& B. J. Wills (Dordrecht: Reidel), IAU Symp., 44, 353

Diaz, J. D., Koposov, S. E., Irwin, M., et al. 2014, MNRAS, 443, 1688

Ekholm, T., Baryshev, Y., Teerikorpi, P., et al. 2001, A\&A, 368, L17

Falco, M., Hansen, S. H., Wojtak, R., et al. 2014, MNRAS, 442, 1887

Fragione, G., \& Loeb, A. 2017, New Astron., 55, 32

Huxor, A. P., Mackey, A. D., Ferguson, A. M. N., et al. 2014, MNRAS, 442, 2165

Ibata, R., Martin, N. F., Irwin, M., et al. 2007, ApJ, 671, 1591

Ibata, R. A., Lewis, G. F., Conn, A. R., et al. 2013, Nature, 493, 62

Ibata, R. A., Lewis, G. F., McConnachie, A. W., et al. 2014, ApJ, 780, 128

Kaisina, E. I., Makarov, D. I., \& Karachentsev, I. D. 2012, Astrophys. Bull., 67, 115

Karachentsev, I. D., \& Kashibadze, O. G. 2006, Astrophysics, 49, 3 
Karachentsev, I. D., \& Kudrya, Y. N. 2014, AJ, 148, 50

Karachentsev, I. D., \& Nasonova, O. G. 2010, MNRAS, 405, 1075

Karachentsev, I. D., Sharina, M. E., Makarov, D. I., et al. 2002, A\&A, 389, 812

Karachentsev, I. D., Dolphin, A. E., Tully, R. B., et al. 2006, AJ, 131, 1361

Karachentsev, I. D., Kashibadze, O. G., Makarov, D. I., \& Tully, R. B. 2009, MNRAS, 393, 1265

Karachentsev, I. D., Makarov, D. I., \& Kaisina, E. I. 2013, AJ, 145, 101

Karachentsev, I. D., Tully, R. B., Wu, Po-Feng, Shaya, E. J., \& Dolphin, A. E. 2014, ApJ, 782, 4

Koposov, S. E., Belokurov, V., Torrealba, G., \& Wyn, E. N. 2015, ApJ, 805, 130

Kourkchi, E., \& Tully, R. B. 2017, ApJ, 843, 16

Kroupa, P. 2014, ArXiv e-prints [arXiv: 1409.6302]

Lynden-Bell, D. 1981, Observatory, 101, 111

Martin, N. F., McConnachie, A. W., Irwin, M., et al. 2009, ApJ, 705, 758

McConnachie, A. W. 2012, AJ, 144, 4

McMillan, P. J. 2017, MNRAS, 465, 76

Nasonova, O. G., de Freitas Pacheco, J. A., \& Karachentsev, I. D. 2011, A\&A, 532, A104

Patel, E., Besla, G., \& Mandel, K. 2017, MNRAS, 468, 3428

Pawlowski, M. S., Famaey, B., Jerjen, H., et al. 2014, MNRAS, 442, 2362
Peebles, P. J. E. 1976, ApJ, 205, 318

Peebles, P. J. E. 2017, ArXiv e-prints [arXiv: 1705 . 10683]

Peirani, S., \& de Freitas Pacheco, J. A. 2008, A\&A, 488, 845

Penarrubia, J., \& Fattahi, A. 2017, MNRAS, 468, 1300

Penarrubia, J., Ma, Y. Z., Walker, M. G., \& McConnachie, A. W. 2014, MNRAS, 443, 2204

Penarrubia, J., Gomez, F. A., Besla, G., et al. 2016, MNRAS, 456, L54

Planck Collaboration XVI. 2014, A\&A, 571, A16

Sandage, A. 1986, ApJ, 307, 1

Sandage, A., \& Tammann, G. A. 1975, ApJ, 196, 313

Shull, J. M. 2014, ApJ, 784, 142

Sofue, Y. 2015, PASJ, 67, 75

Spergel, D. N. 2007, ApJS, 170, 377

Teerikorpi, P., Chernin, A. D., \& Baryshev, Y. V. 2005, A\&A, 440, 791

Tonry, J. L., Stubbs, C. W., Lykke, K. R., et al. 2012, ApJ, 750, 99

Tully, R. B., \& Fisher, J. R. 1977, A\&A, 54, 661

Tully, R. B., \& Shaya, E. J. 1984, ApJ, 281, 31

Tully, R. B., Courtois, H. M., \& Sorce, J. G. 2016, AJ, 152, 50

van der Marel, R. P., Fardal, M., Besla, G., et al. 2012, ApJ, 753, 8

Veljanoski, J., Ferguson, A. M. N., Mackey, A. D., et al. 2013, ApJ, 768, L33 